\title{
AN ASYMPTOTIC ANALYSIS OF LINEAR INTERFACIAL MOTION*
}

\author{
JIN WANG W $^{\dagger}$
}

\begin{abstract}
In this paper, we perform an asymptotic study on slightly viscous flows between two immiscible incompressible fluids. The motion is governed by linearized Navier-Stokes equations together with interfacial conditions. A second-order asymptotic expansion with respect to viscosity is obtained by using the method of multiple scales. In particular, viscous decay rate for the interfacial wave amplitude and viscous correction for the phase speed are explicitly identified.
\end{abstract}

Key words. Navier-Stokes equations, interfacial motion, asymptotic expansion, multiple scales

AMS subject classifications. 76M45 76D05

1. Introduction. Although both viscosities in air and water are very small, they play a crucial role in transferring energy between the two fluids, and directly affect the evolution of water waves. No doubt great success has been achieved in the broad study of viscous flows. However, understanding of viscous effects on interfacial motions, typically in a system of air and water, remains limited due to the physical phenomena implicit in both air flow and water wave evolution. Most of such problems are nonlinear and analytical solutions are impossible. Numerical simulations provide a good way to study the complicated physics involved in those problems [8][14][16]. However, the design of a numerical algorithm which allows an accurate representation of the interface and does not introduce numerical smoothing can be a real challenge [3].

Asymptotic theory offers another effective way to study the viscous flows. In general, this approach applies to problems involving one or more small parameters. Asymptotic methods construct a series expansion, called asymptotic expansion, in terms of the small parameter(s) to approximate the solution. This approximation becomes increasingly accurate when letting the small parameter(s) tend to zero. Probably the two most common asymptotic methods applied to fluid dynamics are the matched asymptotic expansions [18] and the multiple scales [10][12]. The first method constructs the outer and inner solutions for the regions out of and inside the boundary layers, respectively, and these two solutions are then matched to form a single uniformly valid composite solution. The second method introduces one or more independent variables with different scales, known as fast and slow variables, and derive a single asymptotic expansion dependent on these variables. The removal of secularity then ensures the uniformity of the solution.

The nature of the asymptotic theory makes itself an excellent tool for studying fluid flows with small viscosities, such as those in air and water. However, to date few work has ever been published for the asymptotic solutions of viscous interfacial flows. The difficulty stems from the strong coupling between the viscosity and nonlinearity in both the Navier-Stokes equations and interfacial conditions, resulting in the presence of thin boundary layers near the interface in both fluids, which makes the asymptotic expansions extremely complicated.

The strategy we are taking to attack such problems is to start from a simpler case. The linear interfacial motion thus provides a perfect starting point. The present paper

\footnotetext{
*Received April 19, 2006; accepted for publication October 18, 2007.

${ }^{\dagger}$ Department of Mathematics and Statistics, Old Dominion University, Norfolk, VA 23529, USA (j3wang@odu.edu).
} 
is the first in a series of papers to study viscous interfacial flows through asymptotic analysis. In this paper, we perform asymptotic expansions for the linear motion by using the method of multiple scales. Although analytical solutions have already been calculated in the linear case [2][6], the asymptotic solutions can improve our understanding of the influence of viscosity. In particular, the asymptotic study explicitly identifies the viscous decay rate for the amplitude and the viscous correction for the phase speed of the motion. Such information is not revealed from the analytic results since that requires a nonlinear equation, known as the dispersion relation [2][6], to be solved. On the other hand, the analytical results can provide a good validation of the asymptotic expansions. Furthermore, the techniques applied in studying the linear motions and the results found will be used to attack the nonlinear interfacial problems. The forthcoming paper [19] will be concerned with the asymptotic study of viscous effects on nonlinear progressive interfacial waves, especially those with permanent forms (Stokes waves [15][17]).

The outline of the present paper is as follows. First, we present the basic formulation of the two-variable expansions for solutions of two-dimensional linear interfacial flows. Then, we derive the first and second order formal solutions in closed form. As should be clear from our discussion, there is nothing inherently difficult to carry the procedure to even higher order solutions.

2. Asymptotic Formulation. Consider a region of two-dimensional space that contains the upper fluid (air, for example) and the lower fluid (water, for example), which are separated by a sharp interface $h$. Let us denote the spatial coordinates by $(x, z)$, the temporal coordinate by $t$, the velocity components by $(u, w)$, the pressure by $p$, the density by $\rho$, the kinematic viscosity by $\nu$, the gravitational acceleration by $g$ and the surface tension coefficient by $T$. We consider the linearized Navier-Stokes equations:

$$
\begin{gathered}
u_{t}=-\frac{1}{\rho} P_{x}+\nu\left(u_{x x}+u_{z z}\right), \\
w_{t}=-\frac{1}{\rho} P_{z}+\nu\left(w_{x x}+w_{z z}\right), \\
u_{x}+w_{z}=0,
\end{gathered}
$$

where $P$ is the hydrodynamic pressure which includes the gravity term, $P=p+\rho g z$. The motion of the interface is governed by the kinematic condition; other interfacial conditions are determined by the continuity of velocities and the balance of stresses [4]. Specifically,

$$
\begin{aligned}
u^{(1)} & =u^{(2)} \\
h_{t}=w^{(1)} & =w^{(2)} \\
\rho^{(1)} \nu^{(1)}\left(u_{z}^{(1)}+w_{x}^{(1)}\right) & =\rho^{(2)} \nu^{(2)}\left(u_{z}^{(2)}+w_{x}^{(2)}\right) \\
\left(\rho^{(2)}-\rho^{(1)}\right) g h+P^{(1)}-P^{(2)} & -2\left(\rho^{(1)} \nu^{(1)} w_{z}^{(1)}-\rho^{(2)} \nu^{(2)} w_{z}^{(2)}\right)=T h_{x x} .
\end{aligned}
$$

Here and in what follows, we use the superscripts (1) and (2) to distinguish the quantities in the upper and lower fluids but we shall use them only when it is important to distinguish which fluid is being considered.

We assume periodic boundary conditions in the horizontal direction. Meanwhile, solutions are required to decay away from the interface. In order to simplify the calculations, we consider solutions in complex form. Real solutions can be generated by simply adding the complex conjugates. 
Let $k$ be the wave number. For convenience of presentation, $k>0$. We assume solutions take the form

$$
\left(\begin{array}{c}
u \\
w \\
P \\
h
\end{array}\right)=e^{i k x} e^{\sigma t}\left(\begin{array}{c}
\mathcal{U} \\
\mathcal{W} \\
\mathcal{P} \\
a
\end{array}\right)
$$

where $a$ is a fixed number which measures the initial amplitude of the interface. Our goal is then to derive asymptotic expansions for $\mathcal{U}, \mathcal{W}, \mathcal{P}$ and $\sigma$ in terms of small viscosity $\nu$.

By substituting (8) into the equations (1)-(7), we obtain

$$
\begin{gathered}
\sigma \mathcal{U}=-\frac{i k}{\rho} \mathcal{P}+\nu\left(-k^{2} \mathcal{U}+\mathcal{U}_{z z}\right) \\
\sigma \mathcal{W}=-\frac{1}{\rho} \mathcal{P}_{z}+\nu\left(-k^{2} \mathcal{W}+\mathcal{W}_{z z}\right) \\
i k \mathcal{U}+\mathcal{W}_{z}=0
\end{gathered}
$$

and

$$
\begin{aligned}
\mathcal{U}^{(1)} & =\mathcal{U}^{(2)} \\
a \sigma=\mathcal{W}^{(1)} & =\mathcal{W}^{(2)} \\
\rho^{(1)} \nu^{(1)}\left(\mathcal{U}_{z}^{(1)}+i k \mathcal{W}^{(1)}\right) & =\rho^{(2)} \nu^{(2)}\left(\mathcal{U}_{z}^{(2)}+i k \mathcal{W}^{(2)}\right) \\
\left(\rho^{(2)}-\rho^{(1)}\right) g a+\mathcal{P}^{(1)}-\mathcal{P}^{(2)} & -2\left(\rho^{(1)} \nu^{(1)} \mathcal{W}_{z}^{(1)}-\rho^{(2)} \nu^{(2)} \mathcal{W}_{z}^{(2)}\right) \\
& =-k^{2} T a
\end{aligned}
$$

It is convenient to introduce two dimensionless parameters

$$
r=\frac{\rho^{(1)}}{\rho^{(2)}}, \quad R=\sqrt{\frac{\nu^{(2)}}{\nu^{(1)}}} .
$$

In this paper, $r$ and $R$ are constants, which means the ratios of the two densities, and the two viscosites, are fixed. We will use the method of multiple scales [10][12] to derive the asymptotic expansions. In considering the boundary layers near the interface which have thickness proportional to $\sqrt{\nu}$, we introduce scaled vertical coordinates $\eta_{0}, \eta_{1}$ by

$$
\eta_{0}=\frac{z}{\sqrt{\nu}}, \quad \eta_{1}=\sqrt{\nu} \eta_{0}=z
$$

Consequently,

$$
\begin{aligned}
\frac{\partial}{\partial z} & =\frac{1}{\sqrt{\nu}} \frac{\partial}{\partial \eta_{0}}+\frac{\partial}{\partial \eta_{1}}, \\
\frac{\partial^{2}}{\partial z^{2}} & =\frac{1}{\nu} \frac{\partial^{2}}{\partial \eta_{0}^{2}}+\frac{2}{\sqrt{\nu}} \frac{\partial^{2}}{\partial \eta_{0} \partial \eta_{1}}+\frac{\partial^{2}}{\partial \eta_{1}^{2}} .
\end{aligned}
$$

Then we assume the following expansions:

$$
\begin{aligned}
\mathcal{U} & =u_{0}\left(\eta_{0}, \eta_{1}\right)+\sqrt{\nu} u_{1}\left(\eta_{0}, \eta_{1}\right)+\nu u_{2}\left(\eta_{0}, \eta_{1}\right)+\cdots, \\
\mathcal{W} & =w_{0}\left(\eta_{0}, \eta_{1}\right)+\sqrt{\nu} w_{1}\left(\eta_{0}, \eta_{1}\right)+\nu w_{2}\left(\eta_{0}, \eta_{1}\right)+\cdots, \\
\mathcal{P} & =P_{0}\left(\eta_{0}, \eta_{1}\right)+\sqrt{\nu} P_{1}\left(\eta_{0}, \eta_{1}\right)+\nu P_{2}\left(\eta_{0}, \eta_{1}\right)+\cdots, \\
\sigma & =\sigma_{0}+\sqrt{\nu} \sigma_{1}+\nu \sigma_{2}+\cdots .
\end{aligned}
$$


Note that $\sigma$ is the same in the upper and lower fluids but with different expansions. They are related by

$$
\sigma_{0}^{(1)}=\sigma_{0}^{(2)} ; \quad \sigma_{m}^{(1)}=R^{m} \sigma_{m}^{(2)}, \quad m=1,2, \cdots .
$$

By substituting (19) into (9), we obtain

$$
\begin{array}{r}
\left(\sigma_{0}+\sqrt{\nu} \sigma_{1}+\nu \sigma_{2}+\cdots\right) u_{0}+\sqrt{\nu}\left(\sigma_{0}+\sqrt{\nu} \sigma_{1}+\nu \sigma_{2}+\cdots\right) u_{1}+ \\
\nu\left(\sigma_{0}+\sqrt{\nu} \sigma_{1}+\nu \sigma_{2}+\cdots\right) u_{2}+\cdots=-\frac{i k}{\rho} P_{0}-\sqrt{\nu} \frac{i k}{\rho} P_{1}-\nu \frac{i k}{\rho} P_{2}+\cdots \\
+\nu\left(-k^{2} u_{0}-\sqrt{\nu} k^{2} u_{1}-\nu k^{2} u_{2}-\cdots+\frac{1}{\nu} \frac{\partial^{2} u_{0}}{\partial \eta_{0}^{2}}+\frac{2}{\sqrt{\nu}} \frac{\partial^{2} u_{0}}{\partial \eta_{0} \partial \eta_{1}}+\frac{\partial^{2} u_{0}}{\partial \eta_{1}^{2}}+\right. \\
\left.\frac{1}{\sqrt{\nu}} \frac{\partial^{2} u_{1}}{\partial \eta_{0}^{2}}+2 \frac{\partial^{2} u_{1}}{\partial \eta_{0} \partial \eta_{1}}+\sqrt{\nu} \frac{\partial^{2} u_{1}}{\partial \eta_{1}^{2}}+\frac{\partial^{2} u_{2}}{\partial \eta_{0}^{2}}+2 \sqrt{\nu} \frac{\partial^{2} u_{2}}{\partial \eta_{0} \partial \eta_{1}}+\nu \frac{\partial^{2} u_{2}}{\partial \eta_{1}^{2}}+\cdots\right) .
\end{array}
$$

Comparison of the coefficients of $\nu^{n}$ yields,

$$
\begin{array}{ll}
\operatorname{order} \nu^{0}: & \sigma_{0} u_{0}=-\frac{i k}{\rho} P_{0}+\frac{\partial^{2} u_{0}}{\partial \eta_{0}^{2}}, \\
\operatorname{order} \nu^{\frac{1}{2}}: & \sigma_{1} u_{0}+\sigma_{0} u_{1}=-\frac{i k}{\rho} P_{1}+2 \frac{\partial^{2} u_{0}}{\partial \eta_{0} \partial \eta_{1}}+\frac{\partial^{2} u_{1}}{\partial \eta_{0}^{2}}, \\
\text { order } \nu^{1}: \quad & \sigma_{2} u_{0}+\sigma_{1} u_{1}+\sigma_{0} u_{2}=-\frac{i k}{\rho} P_{2}-k^{2} u_{0} \\
& +\frac{\partial^{2} u_{0}}{\partial \eta_{1}^{2}}+2 \frac{\partial^{2} u_{1}}{\partial \eta_{0} \partial \eta_{1}}+\frac{\partial^{2} u_{2}}{\partial \eta_{0}^{2}} .
\end{array}
$$

If we substitute (19) into (10) and equate the coefficients of like powers of $\nu^{n}$, we obtain,

$$
\begin{aligned}
& \operatorname{order} \nu^{-\frac{1}{2}}: \frac{\partial P_{0}}{\partial \eta_{0}}=0, \\
& \text { order } \nu^{0}: \quad \sigma_{0} w_{0}=-\frac{1}{\rho} \frac{\partial P_{0}}{\partial \eta_{1}}-\frac{1}{\rho} \frac{\partial P_{1}}{\partial \eta_{0}}+\frac{\partial^{2} w_{0}}{\partial \eta_{0}^{2}}, \\
& \operatorname{order} \nu^{\frac{1}{2}}: \sigma_{1} w_{0}+\sigma_{0} w_{1}=-\frac{1}{\rho} \frac{\partial P_{1}}{\partial \eta_{1}}-\frac{1}{\rho} \frac{\partial P_{2}}{\partial \eta_{0}}+2 \frac{\partial^{2} w_{0}}{\partial \eta_{0} \partial \eta_{1}}+\frac{\partial^{2} w_{1}}{\partial \eta_{0}^{2}} .
\end{aligned}
$$

Similarly, the substitution of (19) into (11) yields,

$$
\begin{aligned}
& \text { order } \nu^{-\frac{1}{2}}: \frac{\partial w_{0}}{\partial \eta_{0}}=0, \\
& \operatorname{order} \nu^{0}: \quad i k u_{0}+\frac{\partial w_{0}}{\partial \eta_{1}}+\frac{\partial w_{1}}{\partial \eta_{0}}=0, \\
& \text { order } \nu^{\frac{1}{2}}: \quad i k u_{1}+\frac{\partial w_{1}}{\partial \eta_{1}}+\frac{\partial w_{2}}{\partial \eta_{0}}=0 \text {. }
\end{aligned}
$$

We also expand the interfacial conditions (12)-(15). Let $\nu=\nu^{(1)}$. Using (16), the substitution of (19) and (20) into (12) yields,

$$
\begin{array}{ll}
\operatorname{order} \nu^{0}: & u_{0}^{(1)}=u_{0}^{(2)}, \\
\operatorname{order} \nu^{\frac{1}{2}}: & u_{1}^{(1)}=R u_{1}^{(2)} .
\end{array}
$$


Substitution into (13) yields,

$$
\begin{array}{ll}
\operatorname{order} \nu^{0}: & a \sigma_{0}^{(1)}=w_{0}^{(1)}=w_{0}^{(2)}=a \sigma_{0}^{(2)} \\
\operatorname{order} \nu^{\frac{1}{2}}: & a \sigma_{1}^{(1)}=w_{1}^{(1)}=R w_{1}^{(2)}=a R \sigma_{1}^{(2)} .
\end{array}
$$

Substitution into (14) yields,

$$
\begin{aligned}
\text { order } \nu^{-\frac{1}{2}}: & r \frac{\partial u_{0}^{(1)}}{\partial \eta_{0}}=R \frac{\partial u_{0}^{(2)}}{\partial \eta_{0}} \\
\text { order } \nu^{0}: & r\left(\frac{\partial u_{0}}{\partial \eta_{1}}+\frac{\partial u_{1}}{\partial \eta_{0}}+i k w_{0}\right)^{(1)}=R^{2}\left(\frac{\partial u_{0}}{\partial \eta_{1}}+\frac{\partial u_{1}}{\partial \eta_{0}}+i k w_{0}\right)^{(2)} .
\end{aligned}
$$

Finally, substitution into (15) yields,

$$
\begin{array}{ll}
\text { order } \nu^{0}: & \left(\rho^{(2)}-\rho^{(1)}\right) g a+P_{0}^{(1)}-P_{0}^{(2)}=-k^{2} T a, \\
\text { order } \nu^{\frac{1}{2}}: & P_{1}^{(1)}-R P_{1}^{(2)}-2\left(\rho^{(1)} \frac{\partial w_{0}^{(1)}}{\partial \eta_{0}}-R \rho^{(2)} \frac{\partial w_{0}^{(2)}}{\partial \eta_{0}}\right)=0 .
\end{array}
$$

Based on equations (22)-(38), etc., solutions will be determined order by order.

3. First-order solutions. We start the calculation by seeking solutions at the first order, i.e., the solutions to $u_{0}, w_{0}, P_{0}$ and $\sigma_{0}$. Application of secularity conditions in the higher order equations will then be used to determine the additional dependency of the solutions on the scaled variables $\eta_{0}, \eta_{1}$. The interfacial conditions at the lowest orders are applied to determine the coefficients in the solutions. Details are as follows.

Equation (25) implies that $P_{0}$ is independent of $\eta_{0}$, i.e.,

$$
P_{0}=P_{0}\left(\eta_{1}\right)
$$

Equation (28) implies that

$$
w_{0}=w_{0}\left(\eta_{1}\right)
$$

From (22) we obtain

$$
\left(\frac{\partial^{2}}{\partial \eta_{0}^{2}}-\sigma_{0}\right) u_{0}=\frac{i k}{\rho} P_{0}\left(\eta_{1}\right) .
$$

Since we want decaying solutions, (41) yields

$$
u_{0}=\left\{\begin{array}{c}
B_{1}\left(\eta_{1}\right) e^{-\sqrt{\sigma_{0}} \eta_{0}}-\frac{i k}{\rho \sigma_{0}} P_{0}\left(\eta_{1}\right) \text { for } z \geq 0, \\
B_{2}\left(\eta_{1}\right) e^{\sqrt{\sigma_{0}} \eta_{0}}-\frac{i k}{\rho \sigma_{0}} P_{0}\left(\eta_{1}\right) \text { for } z \leq 0,
\end{array}\right.
$$

where $B_{1}\left(\eta_{1}\right)$ and $B_{2}\left(\eta_{1}\right)$ are to be determined, and where the square root of $\sigma_{0}$ must be taken with positive real part, as $\sigma_{0}$ may be complex. The Substitution of (42) and (40) into (29) yields, for $z \geq 0$,

$$
i k B_{1}\left(\eta_{1}\right) e^{-\sqrt{\sigma_{0}} \eta_{0}}+\frac{k^{2}}{\rho \sigma_{0}} P_{0}\left(\eta_{1}\right)+\frac{\mathrm{d} w_{0}\left(\eta_{1}\right)}{\mathrm{d} \eta_{1}}+\frac{\partial w_{1}}{\partial \eta_{0}}=0 .
$$


Elimination of secular terms yields

$$
\frac{k^{2}}{\rho \sigma_{0}} P_{0}\left(\eta_{1}\right)+\frac{\mathrm{d} w_{0}\left(\eta_{1}\right)}{\mathrm{d} \eta_{1}}=0 .
$$

Note that (44) also holds for $z \leq 0$. Meanwhile, substitution of (39) and (40) into (26) yields

$$
\sigma_{0} w_{0}\left(\eta_{1}\right)=-\frac{1}{\rho} \frac{\mathrm{d} P_{0}\left(\eta_{1}\right)}{\mathrm{d} \eta_{1}}-\frac{1}{\rho} \frac{\partial P_{1}}{\partial \eta_{0}} .
$$

To remove the secularity we require

$$
\sigma_{0} w_{0}\left(\eta_{1}\right)=-\frac{1}{\rho} \frac{\mathrm{d} P_{0}\left(\eta_{1}\right)}{\mathrm{d} \eta_{1}} .
$$

By substituting (46) into (44), we obtain

$$
\left(\frac{\mathrm{d}^{2}}{\mathrm{~d} \eta_{1}^{2}}-k^{2}\right) w_{0}=0
$$

which implies

$$
w_{0}= \begin{cases}A_{1} e^{-k \eta_{1}} & \text { for } z \geq 0, \\ A_{2} e^{k \eta_{1}} & \text { for } z \leq 0,\end{cases}
$$

where $A_{1}, A_{2}$ are constants. Equations (44) and (48) show that

$$
P_{0}=-\frac{\rho \sigma_{0}}{k^{2}} \frac{\mathrm{d} w_{0}\left(\eta_{1}\right)}{\mathrm{d} \eta_{1}}=\left\{\begin{aligned}
\frac{\rho^{(1)} \sigma_{0}}{k} A_{1} e^{-k \eta_{1}} & \text { for } z \geq 0 \\
-\frac{\rho^{(2)} \sigma_{0}}{k} A_{2} e^{k \eta_{1}} & \text { for } z \leq 0
\end{aligned}\right.
$$

Equations (42) and (49) show that

$$
u_{0}= \begin{cases}B_{1}\left(\eta_{1}\right) e^{-\sqrt{\sigma_{0}} \eta_{0}}-i A_{1} e^{-k \eta_{1}} & \text { for } z \geq 0 \\ B_{2}\left(\eta_{1}\right) e^{\sqrt{\sigma_{0}} \eta_{0}}+i A_{2} e^{k \eta_{1}} & \text { for } z \leq 0 .\end{cases}
$$

Now we determine the forms of $B_{1}\left(\eta_{1}\right)$ and $B_{2}\left(\eta_{1}\right)$. First notice that (45) and (46) indicate that

$$
P_{1}=P_{1}\left(\eta_{1}\right) .
$$

By substituting (50) into (23) we obtain, for $z \geq 0$,

$$
\left(\frac{\partial^{2}}{\partial \eta_{0}^{2}}-\sigma_{0}\right) u_{1}=\frac{i k}{\rho} P_{1}\left(\eta_{1}\right)+\left(\sigma_{1} B_{1}\left(\eta_{1}\right)+2 \sqrt{\sigma_{0}} \frac{\mathrm{d} B_{1}\left(\eta_{1}\right)}{\mathrm{d} \eta_{1}}\right) e^{-\sqrt{\sigma_{0}} \eta_{0}}-i A_{1} \sigma_{1} e^{-k \eta_{1}} .
$$

The secularity condition requires

$$
\sigma_{1} B_{1}\left(\eta_{1}\right)+2 \sqrt{\sigma_{0}} \frac{\mathrm{d} B_{1}\left(\eta_{1}\right)}{\mathrm{d} \eta_{1}}=0,
$$

which implies

$$
B_{1}\left(\eta_{1}\right)=b_{1} e^{-\frac{\sigma_{1}}{2 \sqrt{\sigma_{0}}} \eta_{1}}
$$


where $b_{1}$ is a constant. Similarly, for $z \leq 0$, we obtain

$$
\sigma_{1} B_{2}\left(\eta_{1}\right)-2 \sqrt{\sigma_{0}} \frac{\mathrm{d} B_{2}\left(\eta_{1}\right)}{\mathrm{d} \eta_{1}}=0
$$

which implies

$$
B_{2}\left(\eta_{1}\right)=b_{2} e^{\frac{\sigma_{1}}{2 \sqrt{\sigma_{0}}}} \eta_{1}
$$

where $b_{2}$ is a constant.

Now the interfacial conditions at the lowest order, (31), (33), (35) and (37), will determine the unknowns $A_{1}, A_{2}, b_{1}, b_{2}$ and $\sigma_{0}$, while $\sigma_{1}$ will be determined by the next-order solutions.

Equation (33) implies that

$$
a \sigma_{0}=A_{1}=A_{2},
$$

where $\sigma_{0}=\sigma_{0}^{(1)}=\sigma_{0}^{(2)}$. By substituting (49) and (57) into (37), we obtain

$$
\sigma_{0}^{2}=-\left(\frac{\rho^{(2)}-\rho^{(1)}}{\rho^{(2)}+\rho^{(1)}} g k+\frac{k^{3} T}{\rho^{(2)}+\rho^{(1)}}\right) \triangleq-F^{2} .
$$

Remark 1. Equation (58) shows that when $r>1$ (i.e., $\rho^{(1)}>\rho^{(2)}$ ), the motion is unstable for all wave numbers $0<k<\sqrt{\left(\rho^{(1)}-\rho^{(2)}\right) g / T}$. This is the well-known Rayleigh-Taylor instability [6]. When $r<1$, the motion is stable and $\sigma_{0}$ is purely imaginary. As should be expected, the value of $\sigma_{0}$ recovers the phase speed for the linear inviscid flow. have

By substituting (54), (56) and (57) into (50) and applying the condition (31), we

$$
b_{1}-i a \sigma_{0}=b_{2}+i a \sigma_{0} .
$$

Meanwhile, (35) implies that

$$
r\left(-\sqrt{\sigma_{0}}\right) b_{1}=R\left(\sqrt{\sigma_{0}}\right) b_{2} .
$$

From (59) and (60), we find

$$
b_{1}=\frac{2 i a R \sigma_{0}}{R+r}, \quad b_{2}=-\frac{2 i a r \sigma_{0}}{R+r} .
$$

Therefore, the solutions at the first order are

$$
\begin{aligned}
& u_{0}=\left\{\begin{array}{rr}
\frac{2 i a R \sigma_{0}}{R+r} e^{-\frac{\sigma_{1}}{2 \sqrt{\sigma_{0}}} \eta_{1}} e^{-\sqrt{\sigma_{0}} \eta_{0}}-i a \sigma_{0} e^{-k \eta_{1}} & \text { for } z \geq 0, \\
-\frac{2 i a r \sigma_{0}}{R+r} e^{\frac{\sigma_{1}}{2 \sqrt{\sigma_{0}}} \eta_{1}} e^{\sqrt{\sigma_{0}} \eta_{0}}+i a \sigma_{0} e^{k \eta_{1}} & \text { for } z \leq 0,
\end{array}\right. \\
& w_{0}=\left\{\begin{array}{lr}
a \sigma_{0} e^{-k \eta_{1}} & \text { for } z \geq 0, \\
a \sigma_{0} e^{k \eta_{1}} & \text { for } z \leq 0,
\end{array}\right. \\
& P_{0}=\left\{\begin{array}{rr}
\frac{\rho^{(1)} a \sigma_{0}^{2}}{k} e^{-k \eta_{1}} & \text { for } z \geq 0, \\
-\frac{\rho^{(2)} a \sigma_{0}^{2}}{k} e^{k \eta_{1}} & \text { for } z \leq 0,
\end{array}\right.
\end{aligned}
$$


where $\sigma_{0}$ is given by (58) and $\sigma_{1}$ will be determined by solutions at the next order.

Remark 2. It appears that the viscosity does not make contributions to the solutions of $w$ and $P$ at the present order, but it does contribute to $u$. There is a boundary layer formed in each fluid for the horizontal velocity starting from the very first order solution.

4. Second-order solutions. Here the second-order solutions refer to $u_{1}, w_{1}$, $P_{1}$ and $\sigma_{1}$. The idea is essentially the same as that in calculating the first-order solutions. The governing equations at the present order and the secularity conditions from equations at the next order determine the forms of the solutions. Then the interfacial conditions at the present order determine the coefficients in the solutions. Here are the details.

For $z \geq 0,(43),(44)$ and (54) imply that

$$
i k b_{1} e^{-\frac{\sigma_{1}}{2 \sqrt{\sigma_{0}}} \eta_{1}} e^{-\sqrt{\sigma_{0}} \eta_{0}}+\frac{\partial w_{1}}{\partial \eta_{0}}=0 .
$$

Hence

$$
w_{1}=\frac{i k b_{1}}{\sqrt{\sigma_{0}}} e^{-\frac{\sigma_{1}}{2 \sqrt{\sigma_{0}}} \eta_{1}} e^{-\sqrt{\sigma_{0}} \eta_{0}}+F_{1}\left(\eta_{1}\right) .
$$

Similarly, for $z \leq 0$ we obtain

$$
w_{1}=-\frac{i k b_{2}}{\sqrt{\sigma_{0}}} e^{\frac{\sigma_{1}}{2 \sqrt{\sigma_{0}}} \eta_{1}} e^{\sqrt{\sigma_{0}} \eta_{0}}+F_{2}\left(\eta_{1}\right) .
$$

Here $b_{1}$ and $b_{2}$ are given in (61) while $F_{1}\left(\eta_{1}\right)$ and $F_{2}\left(\eta_{1}\right)$ are to be determined.

Equations (52), (53) and (57) imply that, for $z \geq 0$,

$$
\left(\frac{\partial^{2}}{\partial \eta_{0}^{2}}-\sigma_{0}\right) u_{1}=\frac{i k}{\rho} P_{1}\left(\eta_{1}\right)-i a \sigma_{0} \sigma_{1} e^{-k \eta_{1}} .
$$

Similarly for $z \leq 0$, we obtain

$$
\left(\frac{\partial^{2}}{\partial \eta_{0}^{2}}-\sigma_{0}\right) u_{1}=\frac{i k}{\rho} P_{1}\left(\eta_{1}\right)+i a \sigma_{0} \sigma_{1} e^{k \eta_{1}} .
$$

Hence

$$
u_{1}= \begin{cases}D_{1}\left(\eta_{1}\right) e^{-\sqrt{\sigma_{0}} \eta_{0}}+\frac{i a \sigma_{0} \sigma_{1} e^{-k \eta_{1}}-\frac{i k}{\rho} P_{1}\left(\eta_{1}\right)}{\sigma_{0}} & \text { for } z \geq 0, \\ D_{2}\left(\eta_{1}\right) e^{\sqrt{\sigma_{0}} \eta_{0}}+\frac{-i a \sigma_{0} \sigma_{1} e^{k \eta_{1}}-\frac{i k}{\rho} P_{1}\left(\eta_{1}\right)}{\sigma_{0}} & \text { for } z \leq 0 .\end{cases}
$$

Substitute (70) and (66) into (30) to obtain, for $z \geq 0$,

$$
\begin{gathered}
i k D_{1}\left(\eta_{1}\right) e^{-\sqrt{\sigma_{0}} \eta_{0}}+\frac{i k b_{1}}{\sqrt{\sigma_{0}}}\left(-\frac{\sigma_{1}}{2 \sqrt{\sigma_{0}}}\right) e^{-\frac{\sigma_{1}}{2 \sqrt{\sigma_{0}}} \eta_{1}} e^{-\sqrt{\sigma_{0}} \eta_{0}}+ \\
{\left[-a k \sigma_{1} e^{-k \eta_{1}}+\frac{k^{2}}{\rho \sigma_{0}} P_{1}\left(\eta_{1}\right)+\frac{\mathrm{d} F_{1}\left(\eta_{1}\right)}{\mathrm{d} \eta_{1}}\right]=-\frac{\partial w_{2}}{\partial \eta_{0}}}
\end{gathered}
$$

To remove the secularity, we must have

$$
-a k \sigma_{1} e^{-k \eta_{1}}+\frac{k^{2}}{\rho \sigma_{0}} P_{1}\left(\eta_{1}\right)+\frac{\mathrm{d} F_{1}\left(\eta_{1}\right)}{\mathrm{d} \eta_{1}}=0 .
$$


Meanwhile, substitution of (51), (63) and (66) into (27) yields, for $z \geq 0$,

$$
a \sigma_{0} \sigma_{1} e^{-k \eta_{1}}+\sigma_{0} F_{1}\left(\eta_{1}\right)=-\frac{1}{\rho} \frac{\mathrm{d} P_{1}\left(\eta_{1}\right)}{\mathrm{d} \eta_{1}}-\frac{1}{\rho} \frac{\partial P_{2}}{\partial \eta_{0}} .
$$

The secularity condition requires

$$
a \sigma_{0} \sigma_{1} e^{-k \eta_{1}}+\sigma_{0} F_{1}\left(\eta_{1}\right)=-\frac{1}{\rho} \frac{\mathrm{d} P_{1}\left(\eta_{1}\right)}{\mathrm{d} \eta_{1}} .
$$

Now by substituting (72) into (74), we obtain

$$
\left(\frac{\mathrm{d}^{2}}{\mathrm{~d} \eta_{1}^{2}}-k^{2}\right) F_{1}=0
$$

which implies

$$
F_{1}\left(\eta_{1}\right)=f_{1} e^{-k \eta_{1}}
$$

where $f_{1}$ is a constant. Similarly for $z \leq 0$, we obtain

$$
\left(\frac{\mathrm{d}^{2}}{\mathrm{~d} \eta_{1}^{2}}-k^{2}\right) F_{2}=0
$$

which implies

$$
F_{2}\left(\eta_{1}\right)=f_{2} e^{k \eta_{1}}
$$

where $f_{2}$ is a constant. Hence,

$$
w_{1}=\left\{\begin{aligned}
\frac{i k b_{1}}{\sqrt{\sigma_{0}}} e^{-\frac{\sigma_{1}}{2 \sqrt{\sigma_{0}}} \eta_{1}} e^{-\sqrt{\sigma_{0}} \eta_{0}}+f_{1} e^{-k \eta_{1}} & \text { for } \quad z \geq 0, \\
-\frac{i k b_{2}}{\sqrt{\sigma_{0}}} e^{\frac{\sigma_{1}}{2 \sqrt{\sigma_{0}}} \eta_{1}} e^{\sqrt{\sigma_{0}} \eta_{0}}+f_{2} e^{k \eta_{1}} & \text { for } \quad z \leq 0 .
\end{aligned}\right.
$$

Consequently, we obtain

$$
P_{1}=\left\{\begin{aligned}
\frac{\rho^{(1)} \sigma_{0}}{k}\left(a \sigma_{1}^{(1)}+f_{1}\right) e^{-k \eta_{1}} & \text { for } \quad z \geq 0 \\
-\frac{\rho^{(2)} \sigma_{0}}{k}\left(a \sigma_{1}^{(2)}+f_{2}\right) e^{k \eta_{1}} & \text { for } \quad z \leq 0 .
\end{aligned}\right.
$$

Remark 3. Equation (79) indicates that viscosity starts making contributions to the vertical velocity from this order. Equation (80), on the other hand, shows no influence of viscosity for the pressure. This pattern is true in general, as is known that the pressure does not have viscous boundary layers in linear motions.

Substitute (80) into (70) to obtain

$$
u_{1}= \begin{cases}D_{1}\left(\eta_{1}\right) e^{-\sqrt{\sigma_{0}} \eta_{0}}-i f_{1} e^{-k \eta_{1}} & \text { for } z \geq 0 \\ D_{2}\left(\eta_{1}\right) e^{\sqrt{\sigma_{0}} \eta_{0}}+i f_{2} e^{k \eta_{1}} & \text { for } z \leq 0\end{cases}
$$

Now we determine the forms of $D_{1}\left(\eta_{1}\right)$ and $D_{2}\left(\eta_{1}\right)$. First notice that (73) and (74) indicate that

$$
P_{2}=P_{2}\left(\eta_{1}\right)
$$


Substitute (62), (81) and (82) into (24) to obtain, for $z \geq 0$,

$$
\begin{gathered}
{\left[\left(\sigma_{2}+k^{2}-\frac{\sigma_{1}^{2}}{4 \sigma_{0}}\right) b_{1} e^{-\frac{\sigma_{1}}{2 \sqrt{\sigma_{0}}} \eta_{1}}+\sigma_{1} D_{1}\left(\eta_{1}\right)+2 \sqrt{\sigma_{0}} \frac{\mathrm{d} D_{1}\left(\eta_{1}\right)}{\mathrm{d} \eta_{1}}\right] e^{-\sqrt{\sigma_{0}} \eta_{0}}} \\
-i\left(a \sigma_{0} \sigma_{2}+f_{1} \sigma_{1}\right) e^{-k \eta_{1}}+\frac{i k}{\rho} P_{2}\left(\eta_{1}\right)=\left(\frac{\partial^{2}}{\partial \eta_{0}^{2}}-\sigma_{0}\right) u_{2} .
\end{gathered}
$$

Elimination of secularity in (83) requires that

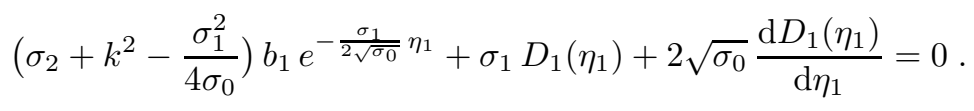

From (84) we can determine the solution for $D_{1}$,

$$
D_{1}\left(\eta_{1}\right)=\left[d_{1}-\frac{b_{1}}{2 \sqrt{\sigma_{0}}}\left(\sigma_{2}+k^{2}-\frac{\sigma_{1}^{2}}{4 \sigma_{0}}\right) \eta_{1}\right] e^{-\frac{\sigma_{1}}{2 \sqrt{\sigma_{0}}} \eta_{1}},
$$

where $b_{1}$ is given in (61) and $d_{1}$ is a constant to be determined. Corresponding to (83), for $z \leq 0$, we obtain

$$
\begin{gathered}
{\left[\left(\sigma_{2}+k^{2}-\frac{\sigma_{1}^{2}}{4 \sigma_{0}}\right) b_{2} e^{\frac{\sigma_{1}}{2 \sqrt{\sigma_{0}}} \eta_{1}}+\sigma_{1} D_{2}\left(\eta_{1}\right)-2 \sqrt{\sigma_{0}} \frac{\mathrm{d} D_{2}\left(\eta_{1}\right)}{\mathrm{d} \eta_{1}}\right] e^{\sqrt{\sigma_{0}} \eta_{0}}} \\
-i\left(a \sigma_{0} \sigma_{2}-f_{2} \sigma_{1}\right) e^{k \eta_{1}}+\frac{i k}{\rho} P_{2}\left(\eta_{1}\right)=\left(\frac{\partial^{2}}{\partial \eta_{0}^{2}}-\sigma_{0}\right) u_{2} .
\end{gathered}
$$

Elimination of secularity of in (86) requires that

$$
\left(\sigma_{2}+k^{2}-\frac{\sigma_{1}^{2}}{4 \sigma_{0}}\right) b_{2} e^{\frac{\sigma_{1}}{2 \sqrt{\sigma_{0}}} \eta_{1}}+\sigma_{1} D_{2}\left(\eta_{1}\right)-2 \sqrt{\sigma_{0}} \frac{\mathrm{d} D_{2}\left(\eta_{1}\right)}{\mathrm{d} \eta_{1}}=0 .
$$

From (87) we can determine the solution for $D_{2}$,

$$
D_{2}\left(\eta_{1}\right)=\left[d_{2}+\frac{b_{2}}{2 \sqrt{\sigma_{0}}}\left(\sigma_{2}+k^{2}-\frac{\sigma_{1}^{2}}{4 \sigma_{0}}\right) \eta_{1}\right] e^{\frac{\sigma_{1}}{2 \sqrt{\sigma_{0}}} \eta_{1}},
$$

where $b_{2}$ is given in (61) and $d_{2}$ is a constant to be determined. We note, in both (85) and (88), that $\eta_{1}$ occurs in the square brackets in the form

$$
\pm \frac{b_{1}}{2 \sqrt{\sigma_{0}}}\left(\sigma_{2}+k^{2}-\frac{\sigma_{1}^{2}}{4 \sigma_{0}}\right) \eta_{1}
$$

and its origin is from the expansion of

$$
\exp \left[ \pm \frac{b_{1}}{2 \sqrt{\sigma_{0}}}\left(\sigma_{2}+k^{2}-\frac{\sigma_{1}^{2}}{4 \sigma_{0}}\right) \sqrt{\nu} \eta_{1}\right] .
$$

This exponential form can be recovered by introducing another scaled coordinate $\eta_{2}=\sqrt{\nu} \eta_{1}=\sqrt{\nu} z$. By following the same procedure as for the determination of $\eta_{1}$ in (85) and (88), the elimination of secularity determines the dependency on $\eta_{2}$.

At this stage, the solutions at the current order are expressed in (79), (80) and (81), with $D_{1}\left(\eta_{1}\right)$ and $D_{2}\left(\eta_{1}\right)$ given by (85) and (88), respectively. What remains is to use the interfacial conditions (32), (34), (36) and (38) to determine the unknown 
coefficients $f_{1}, f_{2}, d_{1}, d_{2}$ and $\sigma_{1}$, while $\sigma_{2}$ has to be determined by solutions at the next order. First, by substituting (79) into (34), we obtain

$$
a \sigma_{1}^{(1)}=\frac{i k b_{1}}{\sqrt{\sigma_{0}}}+f_{1}=-\frac{i k b_{2} R}{\sqrt{\sigma_{0}}}+R f_{2}=a R \sigma_{1}^{(2)} .
$$

Substitution of (61) yields

$$
f_{1}=a \sigma_{1}^{(1)}+\frac{2 a k R \sqrt{\sigma_{0}}}{R+r}, \quad f_{2}=a \sigma_{1}^{(2)}+\frac{2 a k r \sqrt{\sigma_{0}}}{R+r} .
$$

Combine (80) and (91) to obtain

$$
P_{1}=\left\{\begin{aligned}
\frac{\rho^{(1)} \sigma_{0}}{k}\left(2 a \sigma_{1}^{(1)}+\frac{2 a k R \sqrt{\sigma_{0}}}{R+r}\right) e^{-k \eta_{1}} & \text { for } \quad z \geq 0, \\
-\frac{\rho^{(2)} \sigma_{0}}{k}\left(2 a \sigma_{1}^{(2)}+\frac{2 a k r \sqrt{\sigma_{0}}}{R+r}\right) e^{k \eta_{1}} & \text { for } \quad z \leq 0 .
\end{aligned}\right.
$$

By substituting (92) into (38) and recalling (40), we obtain

$$
\sigma_{1}^{(1)}=R \sigma_{1}^{(2)}=-\frac{2 k R r \sqrt{\sigma_{0}}}{(R+r)(1+r)} .
$$

Remark 4. Now the real part of $\sigma_{1}$ is nonzero and it determines the leading term of the decay rate for the wave amplitude due to viscous dissipation, while the imaginary part of $\sigma_{1}$ gives viscous correction to the inviscid phase speed.

Finally, we calculate $d_{1}$ and $d_{2}$. By substituting (81), (85) and (88) into (32) we obtain

$$
d_{1}-i f_{1}=R d_{2}+i R f_{2}
$$

or

$$
d_{1}=R d_{2}+2 i a \sigma_{1}^{(1)}+\frac{2 i a k R \sqrt{\sigma_{0}}}{R+r}(1+r) .
$$

Meanwhile, (36) yields

$$
r\left(2 i a k \sigma_{0}-\sqrt{\sigma_{0}} d_{1}\right)=R^{2}\left(2 i a k \sigma_{0}+\sqrt{\sigma_{0}} d_{2}\right) .
$$

Combine (95) and (96) to obtain

$$
\begin{aligned}
& d_{1}=\frac{1}{R+r}\left\{2 i a R \sigma_{1}^{(1)}+2 i a k \sqrt{\sigma_{0}}\left[R(1+r)+r-R^{2}-\frac{R r(1+r)}{R+r}\right]\right\}, \\
& d_{2}=\frac{1}{R(R+r)}\left\{-2 i a r \sigma_{1}^{(1)}+2 i a k \sqrt{\sigma_{0}}\left[r-R^{2}-\frac{R r(1+r)}{R+r}\right]\right\} .
\end{aligned}
$$

5. Discussion. Except for $\sigma_{2}$, derivation of the second-order asymptotic solutions is complete. This procedure can be certainly continued to find solutions at even higher orders. For example, $\sigma_{2}$ can be found through the calculations at next order:

$$
\sigma_{2}^{(1)}=R^{2} \sigma_{2}^{(2)}=\frac{-2 k^{2}}{(R+r)^{2}(1+r)^{2}}\left[(1+r) R^{4}-2 r^{2} R^{2}+r^{3}(1+r)\right] .
$$


This shows that $\sigma_{2}$ is real and only influences the wave decay rate, and has no contribution for the phase speed. When the viscosity is small, the first few terms in the series expansion, such as

$$
\sigma_{0}+\sqrt{\nu} \sigma_{1}+\nu \sigma_{2}
$$

will give a good estimate for $\sigma$. Otherwise, one has to solve a nonlinear equation of dispersion relation [2] [6], usually by numerical methods, to find an approximate value of $\sigma$. Other flow variables can be determined as well in higher orders, though more complicated calculations will be involved.

It is worth noting that when $r$ is very small (in a system of air and water, for example, $r \doteq 0.001$ ), equations (99) together with (58), (93) and (98) yield a simplified approximation

$$
\sigma \doteq-2 \nu^{(2)} k^{2} \pm i \sqrt{g k+k^{3} T / \rho^{(2)}} .
$$

From (100), it is clear that the wave amplitude will decay exponentially as

$$
a \exp \left[-2 \nu^{(2)} k^{2} t\right]
$$

so that the total energy dissipation rate per wavelength is given by

$$
\frac{d}{d t}\left[\frac{1}{2} \rho^{(2)} k\left(a e^{-2 \nu^{(2)} k^{2} t}\right)^{2} c^{2}\right]=-2 \rho^{(2)} k a^{2} c^{2} e^{-4 \nu^{(2)} k^{2} t},
$$

where $c$ is the phase speed of the wave. This is consistent with the result in Sec. 348 of Lamb's classical book [11].

Although we have treated the ratio of the two viscosities as a constant in this paper, our analysis does allow us to discuss different values of $R$. One special case is $R \rightarrow 0$ (i.e., the viscosity of the upper fluid is relatively much bigger than that of the lower fluid, though both viscosites should be kept small in our framework). From equations (79) - (81), we can observe that $w_{1}^{(1)}$ and $P_{1}^{(1)}$ both approach zero, while $u_{1}^{(1)}$ is nonzero and has a major boundary-layer component. It appears that the horizontal (shear) flow dominates in the upper fluid which has larger viscosity. Similar observation can be made in the lower fluid if we instead let $R$ approach $\infty$.

The asymptotic solutions presented in this paper have been validated by using the analytic results [2]. Hence, they provide a solid background for us to study the nonlinear problems, to which we perform asymptotic expansions in terms of both the viscosity $\nu$ and the wave amplitude $a$. The linear expansions thus provide solutions at the level $a^{1}$, and are used to calculate higher-level solutions in terms of $a^{n}, n=$ $2,3, \cdots$. At each order of $a^{n}$, solutions are expanded with respect to $\nu$ in a form similar to (19); they are determined by solving the nonlinear Navier-Stokes equations together with interfacial conditions, and using lower-order solutions already obtained. The details are given in a separate paper [19].

In addition, the linear analysis presented in this paper can be naturally extended to three-dimensional interfacial motion, especially when periodicity is assumed for the additional horizontal direction, say $y$. In such a case, we seek the solution of $u$ in the form

$$
u=e^{i(k x+l y)} e^{\sigma t} \mathcal{U}
$$

similar forms hold for $w, P$ and $h$. By substituting them into the flow equations, we can derive equations with respect to the vertical coordinate $z$, similar to those in (9)-(15), and then determine solutions order by order. 
Acknowledgment. The author would also like to thank the two anonymous referees for valuable suggestions to improve this paper.

\section{REFERENCES}

[1] Anderson, D. A., Tannehill, J. C. And Pletcher, R. H., Computational fluid mechanics and heat transfer, Hemisphere Publishing Corporation, 1984.

[2] Baker, G. R., Wang, J. And Johnson, J. T., The linear stability at the interface between two immiscible incompressible fluids, preprint.

[3] BakeR, G. R. AND Wang, J., Numerical calculations of viscous effects on water waves, Proceedings of the Third International Conference on Fluid Structure Interaction and Moving Boundary Problems, WIT Press, 2005.

[4] Batchelor, G. K., An introduction to fluid dynamics, Cambridge University Press, 1967.

[5] Billingham, J. And King, A. C., Wave Motion, Cambridge University Press, 2000.

[6] Chandrasekhar, S., Hydrodynamic and Hydromagnetic Stability, Oxford, Clarendon Press, 1961.

[7] Craik, A. D. D., Wave Interactions and Fluid Flows, Cambridge University Press, 1985.

[8] Ferziger, J. H. And Peric, M., Computational Methods for Fluid Dynamics, Springer, 2002.

[9] Gresho, P. M., Incompressible fluid dynamics: Some fundamental formulation issues, Annu. Rev. Fluid Mech., 23 (1991), pp. 413-453.

[10] Hinch, E. J., Perturbation methods, Cambridge University Press, 1991.

[11] LAмB, H., Hydrodynamics, Dover Publications, 1945.

[12] Nayfen, A. H., Perturbation methods, John Wiley \& Sons, 1973.

[13] Ockendon, H. And Ockendon, J. R., Viscous Flow, Cambridge University Press, 1995.

[14] Scardovelli, R. and Zaleski, S., Direct numerical simulation of free surface and interfacial flow, Annu. Rev. Fluid Mech., 31 (1999), pp. 567-603.

[15] Schwartz, L. W. And Fenton, J. D., Strongly Nonlinear Waves, Ann. Rev. Fluid Mech., 14 (1982), pp. 39-60.

[16] Sethinan, J. A., Level set methods and fast marching methods, Cambridge University Press, 2000.

[17] Stokes, G. G., On the theory of oscillatory waves, Trans. Cambridge Philos. Soc., 8 (1847), pp. $441-455$.

[18] Van Dyke, M., Perturbation methods in fluid mechanics, Academic Press, 1964.

[19] Wang, J., An asymptotic expansion for Stokes waves with viscosity, Fluid Dyn. Res., in press.

[20] Whitham, G. B., Linear and Nonlinear Waves, John Wiley \& Sons, 1974. 
J. WANG 